\title{
Comparison of Freeze-fracture- with Cryo-Electron Microscopy on Molecular Assemblies Suitable for Drug \& Gene Delivery
}

\author{
B. Papahadjopoulos-Sternberg \\ NanoAnalytical Laboratory, 3951 Sacramento Street, San Francisco, CA 94118, USA
}

The potency of drug/gene-loaded carriers is frequently depending upon their morphology adopted in a biological relevant environment. Cryofixation techniques such as freeze-fracture electron microscopy (ff-em) and cryo-electron microscopy (cryo-em) are powerful techniques to monitor the self-assembling of lipid- as well as protein-based drug and gene carries.

Cryo-em has a slightly higher resolution compared to ff-em ( $2 \mathrm{~nm}$ for periodical structures) and allows monitoring the inner volume of drug and gene carriers. However, it is restricted to smaller carriers and sub-cellular structures, to low concentrated/viscose dispersions, and has to struggle with beam damage. These drawbacks are irrelevant for ff-em. Ff-em is not only a powerful technique to characterize nanometer and micrometer-size drug/gene carriers but also the method of choice to study their fate related to drug/gene load, application milieu, and during interaction with cells [1-3]. Furthermore it allows distinguishing between bilayer and non-bilayer structures.

Using ff-em we studied the morphology of a wide variety of drug and gene carriers such as multilamellar liposomes (MLV), lipid-stabilized gas bubbles, depofoam particles, cochleate cylinder, niosomes, small unilamellar liposomes (SUV), micelles, and cationic liposome/DNA complexes (CLDC) [1-4]. Because of their small size, spherical micelles $(<50 \mathrm{~nm})$ and small liposomes $(<100 \mathrm{~nm})$ accumulate in pathological areas and are excellent carriers for poorly watersoluble drugs $[5,6]$. Depending upon helper lipid, ionic strength, and gene component CLDC adopt polymorph structures such as spaghetti/ meatballs, map-pins, as well as honeycomb structures $[7,8]$. Parallel studies of transfection activity and morphology of CLDC showed that lipid precipitates displaying honeycomb structure are associated with high transfection rates under in vitro conditions. In vivo transfection activity seems to be associated with small complexes such as map-pin structure [8].

Furthermore the fracture behavior of lipid-based macromolecular assemblies indicates their adoption of bilayer or non-bilayer structures. While bilayer vesicles such as liposomes display convex and concave fracture faces, monolayer-coated gas bubbles show concave, and hard-core particles such as micelles convex fracture faces respectively.

\section{References}

[1] B. Sternberg, Liposome Technology, CRC Press I (1992) 363.

[2] B. Sternberg, Handbook Nonmedical Applications of Liposomes CRC Press (1996) 271.

[3] B. Sternberg, Medical Applications of Liposomes, Elsevier (1998) 395.

[4] B. Sternberg et al., Nature 378 (1995) 21.

[5] V. P. Torchilin et al. PNAS (2003) 100 (4) 1972.

[6] V. P. Torchilin et al. PNAS (2003) 100 (10) 603.

[7] B. Sternberg et al., FEBS-Letters 356 (1994) 361.

[8] B. Sternberg et al., BBA 1375 (1998) 1375. 

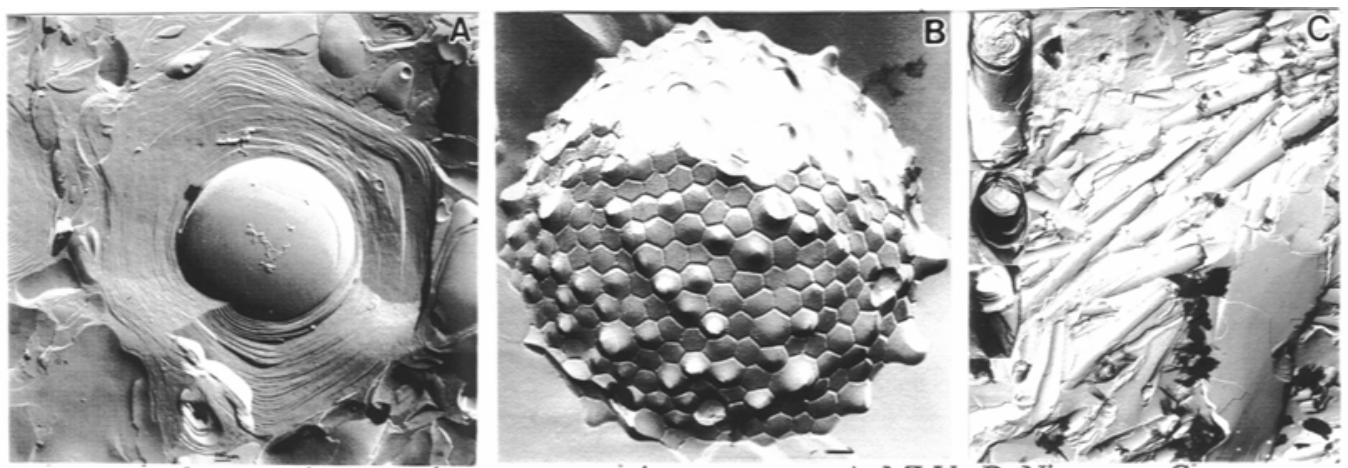

Fig.1. Freeze-fracture electron microscopy on microstructures. A. MLV. B. Niosome. C, Cochleate cylinder. Bar scale represents $100 \mathrm{~nm}$.
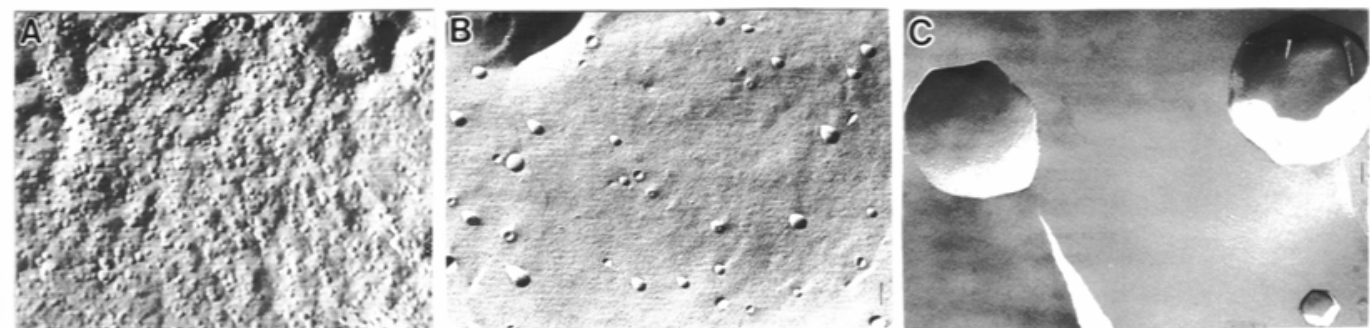

Fig.2. Fracture behavior. A. Convex fracture planes of hard-core particles, B. Concave and convex fracture planes of SUV, C. Concave fracture faces of lipid coated gas bubbles. Bar scale=100nm.
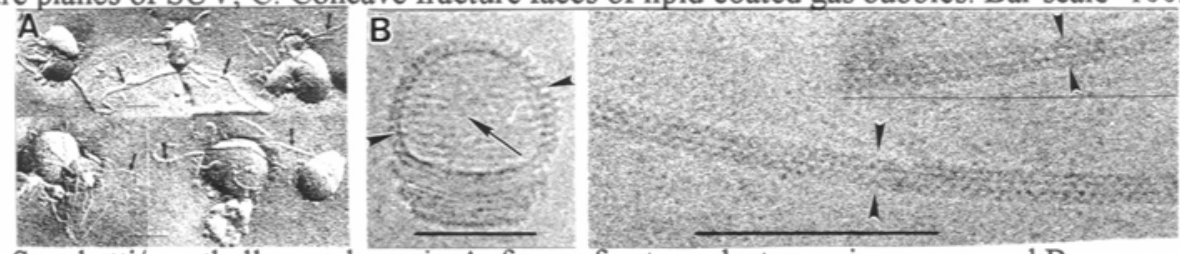

Fig.3. Spaghetti/meatball complexes in A. freeze-fracture electron microscopy and B. cryo-em.

Bar scale $=100 \mathrm{~nm}$. Fig.3B. courtesy of Christoph Böttcher.


Fig.4. Cell interaction of A. Cochleate cylinder, B. SUV, C. spaghetti-meatball structure.

Fig. 4 A +4 B. bar scale $=1 \mu \mathrm{m}$. Fig. 4 C . bare scale $=100 \mathrm{~nm}$. 